\title{
Endoscopic Therapy of Colonic Liver Flexure Mucocele
}

\author{
Xin-Bo Ai ${ }^{a} \quad$ Jia-Cheng Feng ${ }^{b}$ Fei-Yue Gong ${ }^{a} \quad$ An Wang $^{a}$ \\ Da-Hong Ren ${ }^{b}$ Kang Suic
}

Departments of a Gastroenterology, bPathology and ' General Surgery, Third Affiliated Hospital of Jinan University College of Medicine, Zhuhai People's Hospital, Zhuhai, China

\section{Key Words}

Mucocele $\cdot$ Colonic liver flexure $\cdot$ Endoscopic polypectomy

\begin{abstract}
Colorectal mucoceles usually arise in the appendix, and colonic disease is very rare. We report the first case of a mucocele of the colonic liver flexure that was treated successfully with endoscopy. A 36-year-old man was admitted to our hospital because of abdominal distension persisting for 3 days. Colonoscopic examination revealed a round polyp in the hepatic flexure, and we performed hot snare polypectomy with argon plasma coagulation. Histologically, the polypectomy specimen was confirmed to be a mucocele, with no neoplastic changes. Follow-up examinations at 6 and 12 months showed no evidence of recurrence.
\end{abstract}

\section{Introduction}

Mucoceles of the gastrointestinal tract are associated most commonly with the appendix [1]. Mucocele of the appendix is an uncommon disorder which is often asymptomatic but sometimes may cause acute appendicitis-like symptoms. Sometimes patients with mucocele present with confusing symptoms such as abdominal pain, abdominal distension, vomiting and so on [2]. According to the published literature, the etiology of these mucoceles can be divided into four subtypes on the basis of the origin of the epithelial lining of the appendix: (1) simple mucocele attributed to outflow obstruction, (2) mucosal hyperplasia, (3) mucinous cystadenoma, and (4) malignant mucinous cystadenoma [3]. Some authors prefer the term mucocele since most of these lesions are not true cysts due to the absence of epithelial lining [4]. The mechanism of formation of the mucocele is still not completely clear; however, a traumatic etiology rather than an obstructive phenomenon is favored. Bhaskar et al. showed that the escape of mucus into the surrounding tissue after severing the excretory salivary ducts led to 
the formation of mucocele [5]. Preoperative diagnosis for appendiceal mucoceles is very difficult, it needs to be confirmed after surgery. All appendiceal mucoceles should probably be removed to eliminate the chance of progression to malignancy, especially for those measuring at least $2 \mathrm{~cm}[6]$. However, colonic mucoceles are very rare and their medical treatment is still unknown. We searched the key words 'colorectal mucoceles' in PubMed, however, we only found more than 16 results of appendiceal mucoceles. Thus we report the first case of mucocele of the colonic liver flexure treated by endoscopic therapy; there are no findings of recurrence or malignancy at 1-year follow-up.

\section{Case Report}

A 36-year-old male patient was admitted to our hospital for abdominal distension for 3 days. Three days earlier, the patient presented to our hospital for intermittent abdominal distension aggravated after lunch, no fever or chills, no abdominal tenderness and no melena. Physical examination showed: blood pressure $120 / 70 \mathrm{~mm} \mathrm{Hg}$, heart rate $76 \mathrm{bpm}$, no jaundice, no abdominal tenderness, negative shifting sign, bowel sound $4 \mathrm{bpm}$, no edema of the lower limbs. Liver function test: alanine aminotransferase $30 \mathrm{U} / \mathrm{l}$ (normal 0-40 U/l), aspartate aminotransferase $40 \mathrm{U} / \mathrm{l}$ (normal 0-40 U/l), total bilirubin $30.2 \mu \mathrm{mol} / \mathrm{l}$. Serum tumor markers such as carcinoembryonic antigen (CEA), cancer antigen 19-9), cancer antigen 125) and alpha-fetoprotein were within normal range.

Colonoscopic evaluation (CF 260, Olympus, Tokyo, Japan) revealed a round-like polyp (Isp type) located at the liver flexure approximately $1.0 \times 0.9 \mathrm{~cm}$ in size. Gentle probing with a biopsy forceps indicated positive 'pillow sign', and no ulcerations were found on the surface of the polyp. We attempted to lift the lesion by submucosal injection of saline to estimate whether it was a submucosal tumor. Positive nonlifting sign was observed and the lesion could be resected without deeper infiltration (fig. 1). Hot snare polypectomy was performed, blended current with $25 \mathrm{~W}$ was used for polypectomy, and yellow fluid was present after polypectomy. Hemoclip was used to avoid postpolypectomy bleeding (fig. 2), and prior to endoscopic therapy, informed consent was signed. There was no evidence of immediate or delayed complications including bleeding or perforation. Complete resection was defined as complete removal of the mucosal lesion according to endoscopic and pathologic assessment and negative biopsy findings on one follow-up examination [7]. Mucocele of liver flexure was diagnosed by the pathologist and negative margin of the resected lesion was confirmed (fig. 3 ). Histopathological findings indicated no hyperplasia of the crypt epithelium, mucinous cystadenoma or mucinous cystadenocarcinoma. p53, CEA, PCNA and Ki-67 (Shanghai DoBio Biotec Co. Ltd., China) were used for analysis; expression of p53, CEA, PCNA and Ki-67 was negative with immunohistochemistry (fig. 4).

\section{Discussion}

Colonic mucocele of the liver flexure is a very rare disease; we searched the title 'mucoceles of colonic liver flexure' in PubMed and Medline and found no previous publications. Ruiz-Tovar et al. reported that the possible pathogenesis might be mucin retention [8]. With respect to the etiology of colonic mucocele, we presume that it is mainly due to outflow obstruction of mucin. When it comes to its management, Stocchi et al. reported that all mucoceles greater than $2 \mathrm{~cm}$ should be removed surgically [6]. We performed endoscopic therapy due to indication of polypectomy, and no malignancy of the colon mucocele was found according to our histopathological findings. In our case, the patient underwent polypectomy to eliminate the risk of malignancy although it was less than $2 \mathrm{~cm}$. 
In conclusion, we report a case of colonic liver flexure mucocele; the lesion was successfully removed with polypectomy. Moreover, routine colonoscopy was performed with no findings of endoscopic recurrence in the follow-ups at 6 and 12 months, and the patient's symptoms improved significantly after polypectomy.

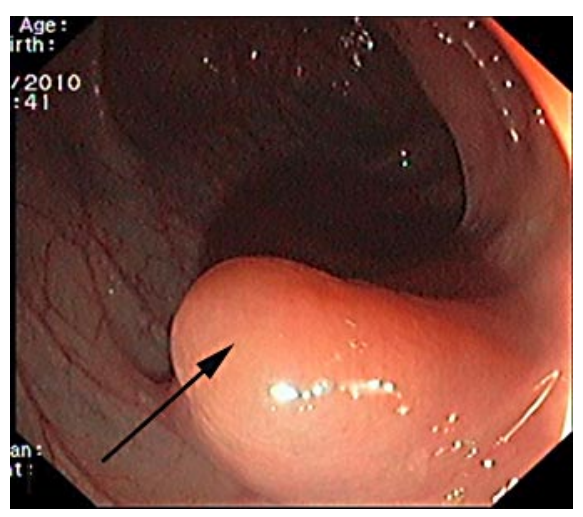

Fig. 1. Colonoscopy showing mucocele of the colonic liver flexure (Isp type) approximately $1.0 \times 0.9 \mathrm{~cm}$ in size (arrow).

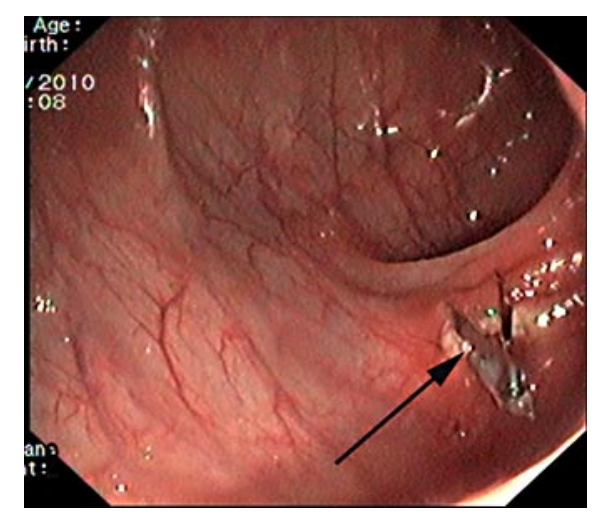

Fig. 2. Colonoscopy showing hemoclips fixed to the bottom of the polyp after polypectomy (arrow). 


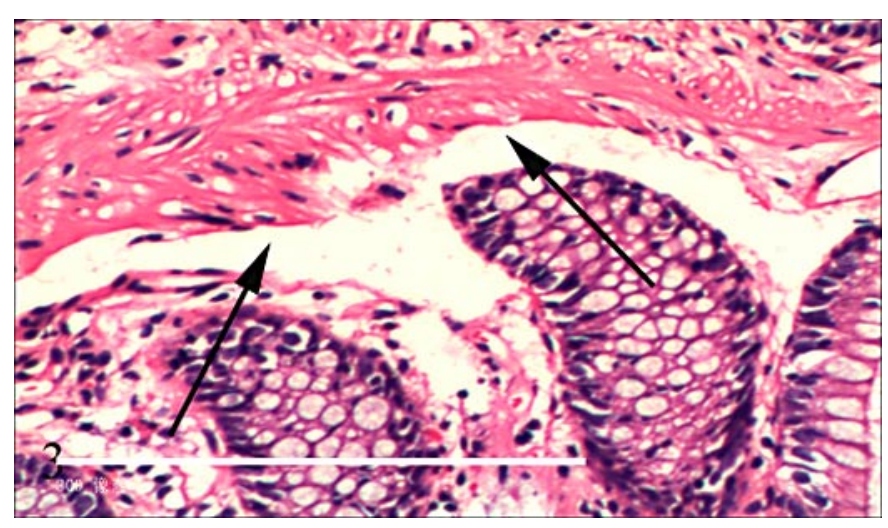

Fig. 3. HE staining showing accumulation of mucin and formation of cyst with inflammatory cells infiltration (arrows), $\times 200$.

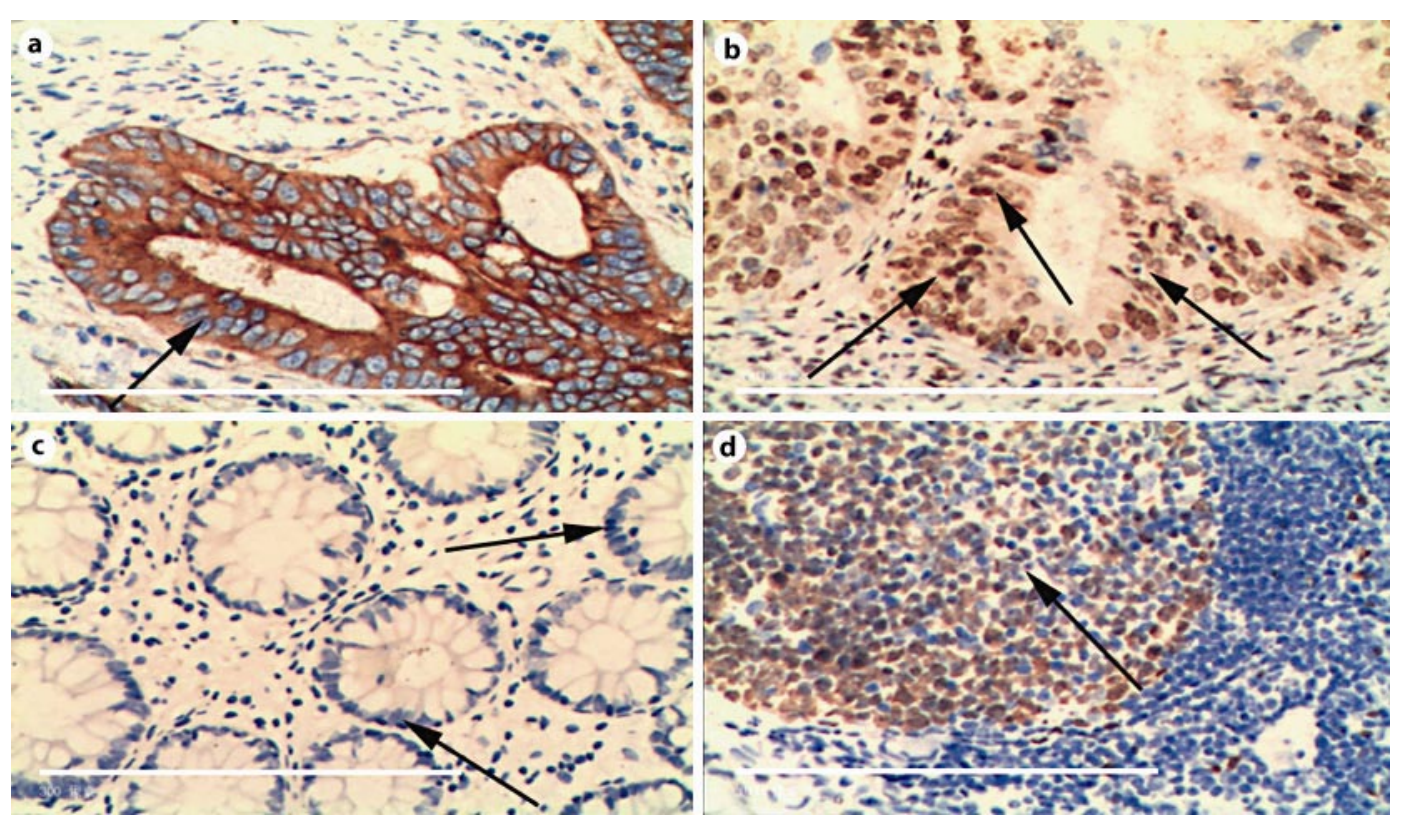

Fig. 4. Expression of p53, CEA, PCNA and Ki-67 with immunohistochemistry. Negative expression of p53, CEA, PCNA and Ki-67 indicated that there was no malignancy or hyperplasia of the mucocele. a Negative for $\mathrm{p} 53$ of epithelial nuclear cells, $\times 200$ (arrow). b Negative for CEA of epithelial nuclear cells, $\times 200$ (arrows). c Negative for PCNA of epithelial cells, $\times 200$ (arrows). d Negative for Ki-67 of lymphatic follicles, $\times 200$ (arrow). 


\section{References}

1 Soweid AM, Clarkston WK, Andrus CH, et al: Diagnosis and management of appendiceal mucoceles. Dig Dis 1998;16:183-186.

-2 Karakaya K, Barut F, Emre AU, et al: Appendiceal mucocele: case reports and review of current literature. World J Gastroenterol 2008;14:2280-2283.

-3 Higa E, Rosai J, Pizzimbono CA, et al: Mucosal hyperplasia, mucinous cystadenoma, and mucinous cystadenocarcinoma of the appendix. A re-evaluation of appendiceal 'mucocele'. Cancer 1973;32:1525-1541.

4 Jornet PL: Labial mucocele: a study of eighteen cases. Int J Dent Sci 2006;3:2.

5 Bhaskar SN, Bolden TE, Weinmann JP: Pathogenesis of mucoceles. J Dent Res 1956;35:863-874.

6 Stocchi L, Wolff BG, Larson DR, et al: Surgical treatment of appendiceal mucocele. Arch Surg 2003;138: 585-589.

7 Ahmad NA, Kochman ML, Long WB, et al: Efficacy, safety, and clinical outcomes of endoscopic mucosal resection: a study of 101 cases. Gastrointest Endosc 2002;55:390-396.

8 Ruiz-Tovar J, Teruel DG, Castiñeiras VM, et al: Mucocele of the appendix. World J Surg 2007;31:542-548. 